\title{
La sialométaplasie nécrosante : revue systématique de la littérature
}

\author{
Sarah Aubies-Trouilh, Jean-Christophe Fricain \\ Université Segalen, Bordeaux, France
}

(Reçu le 17 juillet 2012, accepté le 18 septembre 2012)

Mots clés :

sialométaplasie

nécrosante / glandes

salivaires accessoires

Key words:

necrotizing

sialometaplasia /

minor salivary glands
Résumé - Objectif : Répertorier les caractéristiques épidémiologiques, étiopathogéniques, cliniques, histologiques et thérapeutiques de la sialométaplasie nécrosante (SMN).

Matériel et méthodes : 204 cas répertoriés à partir d'une revue systématique de la littérature.

Résultats : La SMN est une affection bénigne et rare des glandes salivaires. Classiquement, elle se développe à partir des glandes salivaires du palais dur. Survenant essentiellement chez l'adulte, la SMN se révèle le plus souvent par une ulcération palatine cratériforme, bien limitée. D'étiopathogénie incertaine, elle évolue spontanément vers la guérison. Conclusion : L'intérêt de sa connaissance réside dans le risque de confusion clinique et histologique avec certaines lésions néoplasiques pouvant conduire à des traitements radicaux délabrants.

Abstract - Necrotizing sialometaplasia: a systematic review of literature. Objective: To make a list of the epidemiological, etiological, clinical, histological and therapeutic features of necrotizing sialometaplasia (NSM). Material and methods: Analysis of 204 cases from a systematic review of the literature.

Results: NSM is an uncommon benign disease of salivary glands. Usually, NSM affects the minor salivary glands of the hard palate. It occurs primarily in adults, NSM is manifested by a sharply demarcated crater-like palatal ulcer. Although the etiology is uncertain, the lesion heals spontaneously.

Conclusion: Dental surgeon has to know this lesion because of the risk of histological and clinical confusion with malignant lesions which may lead to mutilating radical treatment.
La sialométaplasie nécrosante (SMN) est une maladie inflammatoire rare des glandes salivaires, dont la prévalence est estimée à $0,03 \%$ des lésions de la muqueuse buccale biopsiées [1]. La SMN a été identifiée récemment. En 1958, Saunders [2] a rapporté, dans une revue générale sur les stomatites nicotiniques du palais, deux observations cliniques très évocatrices d'une SMN palatine. Cette entité a été individualisée pour la première fois en 1973 par Abrams et al. [3] à partir de sept observations de localisation palatine. En 1974, Dunlap et al. [4] ont rapportés cinq nouveaux cas. En France, les premières publications sur la sialométaplasie nécrosante datent de 1979 [5].

Depuis, peu d'études ont été publiées, en dehors de petites séries dont il est difficile de tirer des conclusions. La revue de la littérature la plus récente a été publiée en 1991 [6]. L'objectif de cette revue systématique était de répertorier les caractéristiques épidémiologiques, étiologiques, cliniques, histologiques et thérapeutiques de la SMN.

\section{Matériel et méthodes}

La recherche a été effectuée sur les quarante dernières années. Les mots clés indexés dans le MeSH « necrotizing sialometaplasia » et « minor salivary glands » ont été appliqués dans trois bases de données: Pascal (36 références), Scopus (244 références), Pubmed (230 références). Les langues de recherche ont été l'anglais, l'espagnol, l'italien, le portugais et le français.

143 articles ont été sélectionnés, il s'agissait des articles disponibles dans les bibliothèques de l'université Bordeaux Segalen et la bibliothèque inter-universitaire de santé de Paris.

\footnotetext{
*Correspondance : sarah.aubies@hotmail.fr, fricainj@aol.com
} 
Tableau I. Age et sexe en fonction des différentes localisations de la SMN.

Table I. Age and sex according to the NSM's different localisations.

\begin{tabular}{lccccc}
\hline & \multicolumn{2}{c}{ AGE MOYEN (ans) } & \multicolumn{2}{c}{ POURCENTAGE } \\
\hline LOCALISATION & Hommes et Femmes & Hommes & Femmes & Hommes & Femmes \\
\hline Glandes salivaires accessoires & 45 & 47 & 41 & $58 \%$ & $42 \%$ \\
Glandes salivaires principales & 53 & 53 & 53 & $44 \%$ & $56 \%$ \\
Toutes les localisations & 46 & 48 & 43 & $56 \%$ \\
\hline
\end{tabular}

Sexe : - Homme : $[3,4,7-9,11,14-17,19-23,25-46,48,50,51,53,55-59,61,63,64,66-69,71-73,75,76,82,86-88,90,92-95,97$, $98,102,104,109,110,113]$

- Femme : $[3-5,7,8,10,12,13,18,20,21,23-25,27-29,31,35,41-43,47,49,50,52,54,57,60,62,65,70,74,77-81,83-85$, $89-91,93,96,99,100,101,103,105-107,111,112,114-117]$

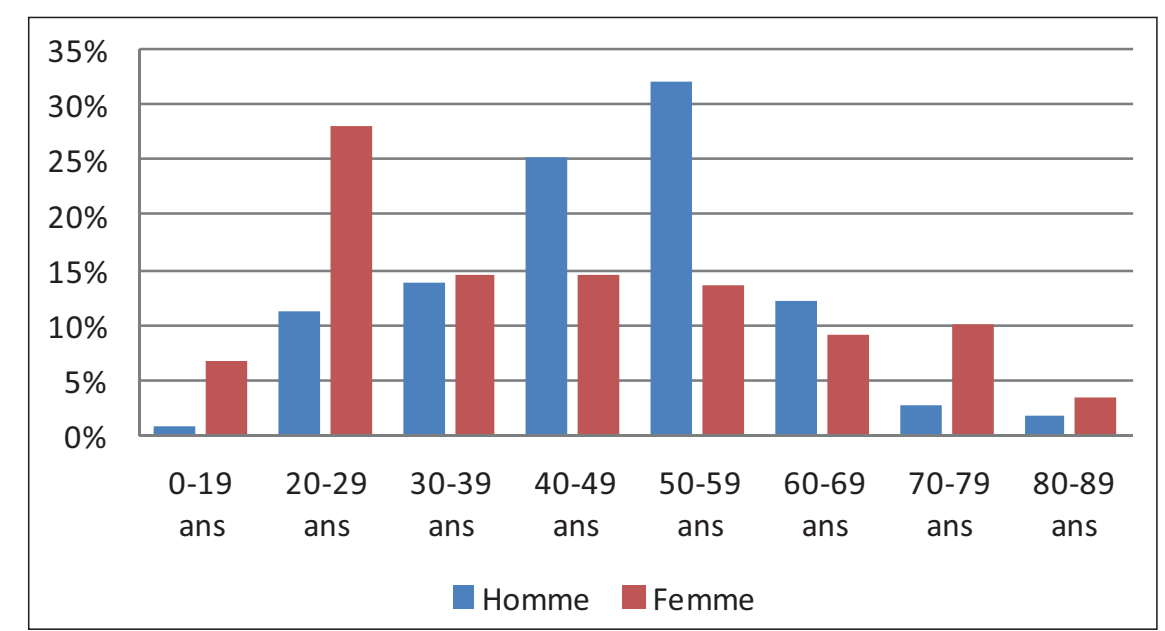

Fig. 1. Répartition des SMN en fonction de l'âge et du sexe.

Fig. 1. NSM's distribution according to age and sex.

Age :

- $0-19$ ans : $[10,20,23,31,54,59,111]$

- $\quad 20-29$ ans : $[3,4,8,21,23,24,28,29,41,43,49,50,51,55,62,70,76-78,80,85,91,93,96,98,101-103,106]$

- $\quad 30-39$ ans : $[3,5,7,10,21,23,24,35,36,41,42,57,58,66,68,70,71,74,79,81,84,86,89,90,92,93,108,113]$

- $40-49$ ans : $[3,4,7,14,18,20,21,23,25-30,38,40-42,44,45,50,52,57,61,64,65,70,75,82,83,87,88,95,98,105]$

- $50-59$ ans : $[3,7-10,12,13,15,17,20-23,25,28,31,33,34,37,39,41-43,46,48,57,61,63,67,72,73,90,97,100,104,107$, $112,114,115]$

- $60-69$ ans : $[4,8,10,16,19,20,23,25,27-29,32,35,36,40,43,69,94,99,109]$

- $70-79$ ans : $[10,25,29,42,43,56,117,116]$

- 80-89 ans : [11, 23, 47, 53, 60]

114 articles décrivant des cas originaux ont été utilisés pour la revue systématique qui a permis de répertorier 204 cas de SMN.

\section{Résultats}

\section{Epidémiologie}

$88 \%$ des cas de SMN intéressaient les glandes salivaires accessoires et $12 \%$ des cas les glandes salivaires principales ; $79 \%$ des cas étaient localisés au palais.
Pour les glandes salivaires accessoires et principales, $56 \%$ des cas étaient de sexe masculin, ce qui correspondait à un sex-ratio de 1,3. Ce rapport était inversé pour les glandes salivaires principales (Tab. I).

La SMN a été rapportée chez des patients âgés de 2 à 87 ans. L'âge moyen est de 46 ans. La SMN est apparue dans $24 \%$ des cas entre 50 et 59 ans, et dans $21 \%$ des cas entre 40 et 49 ans. Dans ces deux tranches d'âge, on note une prédominance masculine. Pour les tranches d'âge antérieures, les femmes sont plus atteintes que les hommes (Fig. 1). 


\section{Etiopathogénie}

L'étiopathogénie de la SMN n'est pas connue avec certitude mais de nombreuses théories ont été suggérées.

\section{Théorie vasculaire}

Il existe un consensus général selon lequel la lésion serait précédée par un évènement ischémique. Selon cette théorie, suggérée en premier par Abrams et al. [3], tout agent (physique, chimique ou infectieux) capable de causer une tuméfaction locale pourrait entraîner un déficit vasculaire local et conduire à une ischémie responsable de la nécrose des lobules salivaires. La réparation s'exprimerait par une métaplasie épidermoïde et une ré-épithélialisation de la zone ulcérée [7].

La SMN peut survenir sur un terrain vasculaire : une athérosclérose [8], une hypertension artérielle [8-16], une maladie de Buerger [17]. Dans d'autres cas, un facteur traumatique (physique, chimique, thermique...) précédant la survenue de la SMN a été retrouvé : injections d'anesthésiques locaux et usage de produits chimiques lors des soins dentaires [15, 1723], ingestion de boissons chaudes [24], port de prothèses dentaires $[3,8-11,18,21,23,25-39]$, intervention chirurgicale loco-régionale [18-23, 40-54]. Quelquefois, une tumeur des voies aéro-digestives supérieures susceptible de créer des modifications vasculaires loco-régionales était associée à la SMN $[8,10,16,23,28,38,40-43,45-48,50,53,55-61]$.

\section{Autres théories}

\section{Théorie de l'intoxication tabagique}

La notion d'une intoxication tabagique est souvent mentionnée. Dans la revue systématique de la littérature, on retrouve $30 \%$ de fumeurs $[3,7-9,14,17,18,24,25,28,29$, $32,34,37,39,40,45,49,57,62-87]$. La SMN représenterait le stade terminal ou ulcératif $d^{\prime}$ une stomatite nicotinique palatine [73].

\section{Théorie carentielle}

Le rôle d'une carence en vitamine $A$, responsable d'une métaplasie malpighienne des canaux a été évoqué [29].

\section{Théorie allergique}

En raison de la présence de polynucléaires éosinophiles dans l'infiltrat inflammatoire de la SMN, un mécanisme d'origine allergique a été proposé $[49,68]$. Un cas faisant suite à un eczéma de contact à la néomycine a été rapporté [47]. $3 \%$ des cas présentaient des allergies, médicamenteuses ou non $[12,47,49,80,83,88]$.

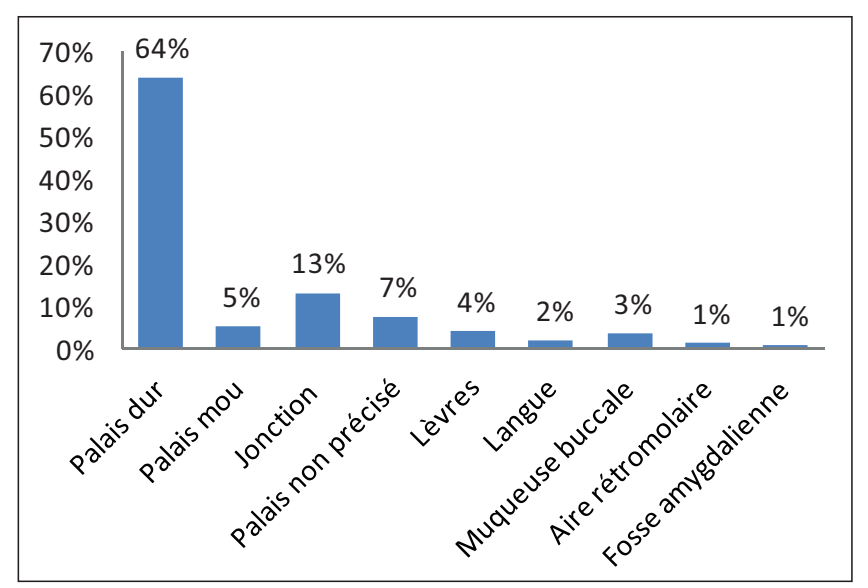

Fig. 2. Répartition des différentes localisations de la SMN dans les glandes salivaires accessoires.

Fig. 2. Distribution of the NSM's different localisations in the minor salivary glands.

\section{Théorie infectieuse}

Dans $5 \%$ des cas, on retrouve une infection ORL récente $[9,13-15,54,61,70,74,89,90]$. Aucun agent pathogène n'a été mis en évidence $[9,24,69,91]$ à l'exception de deux cas qui signalaient la présence de Candida albicans [30, 92]. Des cas de SMN ont été décrits chez des patients porteurs du VIH $[39,86]$.

\section{Théorie de l'anorexie et de la boulimie}

Huit cas survenant chez des jeunes femmes anorexiques et/ ou boulimiques ont été répertoriés [10, 24, 78, 80, 81].

\section{Description et diagnostic cliniques}

SMN des glandes salivaires accessoires

\section{Localisation}

$79 \%$ des cas de SMN ont un siège palatin. Ils se répartissent, par ordre décroissant, sur le palais dur [3-5, 7-10, 12, 13-15, 17-22, 24-29, 32, 34-41, 44, 49, 55, 57, 58, 61-65, 69, $70,73-78,80-88,90,91,93-103]$, à la jonction entre le palais dur et le palais mou $[11,20,25,30,31,33,35,37,41,56$, $57,68,70,79,89,92,93,104-106]$ et sur le palais mou [4, $10,21,28,40,48,54,66,72,107] .13$ cas sont non documentés $[4,20,23,24,41,50,67]$.

$9 \%$ des cas de SMN sont survenus sur des sites buccaux autres que le palais : lèvres $[23,47,50,51,71]$, muqueuse buccale $[23,28,31,45,53,108]$, langue $[23,52,60]$, zone rétromolaire [109, 110] et loge amygdalienne [59] (Fig. 2). 


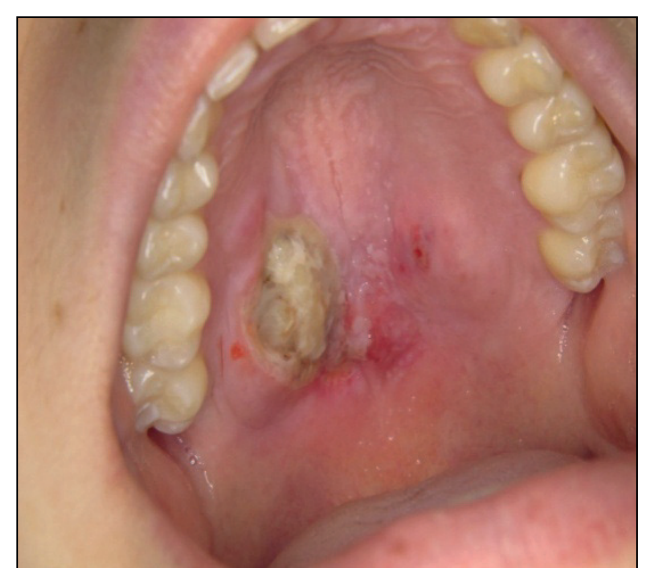

Fig. 3a. SMN palatine bilatérale : ulcération et tuméfaction. Fig. 3a. Bilateral palatal NSM: ulcer and mass.

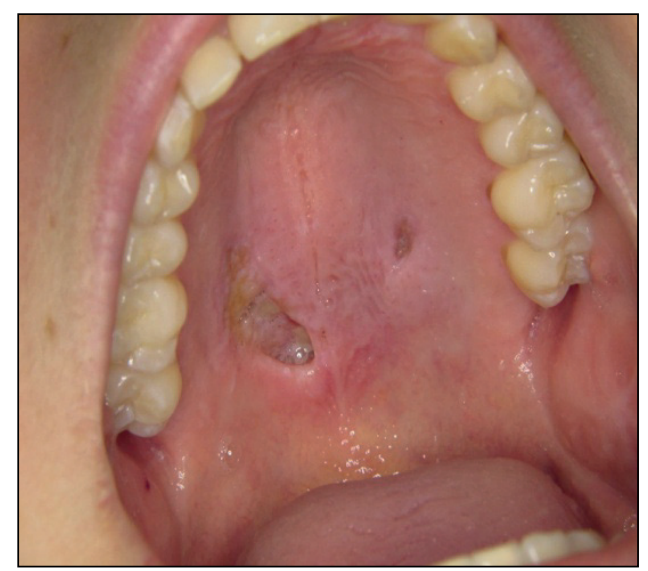

Fig. 3b. SMN palatine bilatérale 1 mois après la Figure 3 a. Fig. 3b. Bilateral palatal NSM one month after the Figure $3 a$.

\section{Lésion élémentaire}

Dans $61 \%$ des cas palatins $[3-5,7-13,15,17-23,25-27$, $29,30,32,34-41,55,56,61,64-68,70,74-76,78-80,83$, $84,86-88,90,92,93,97-100,102,104-107]$, elle réalisait une ulcération profonde, d'aspect cratériforme, aux bords nets parfois surélevés (Fig. 3a). Le fond était nécrotique et l'ulcération pouvait être cernée par un halo érythémateux [3, 64].

Dans $79 \%$ des cas palatins $[3-5,7-11,13,14,15,17,18$, 20-37, 39, 41, 44, 48-50, 54, 55, 57, 61, 63, 64, 66, 70, 72$79,81,82,84-88,90,93-95,97-105,107]$, il s'agissait d'une lésion unilatérale (Figs. 4 et 6 ), dans $19 \%$ des cas de lésions bilatérales $[4,8,12,19,20,24,29,35,38,40,41,56-58,61$, $62,65,67-70,80,83,89,91,96,98]$ (Fig. 3b) et dans $2 \%$ des cas il y avait plus de 2 lésions $[21,92,106]$. Dans $16 \%$ des cas palatins $[8,10,14,24,29,33,41,62,63,69,70,72$, $73,77,81,85,91,96,98,101]$, l'ulcération faisait suite à une tuméfaction rouge violacée du palais (Fig. 4).

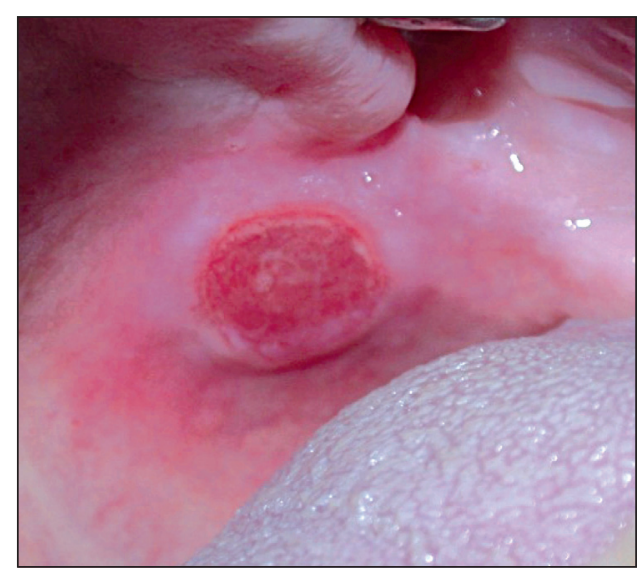

Fig. 4. SMN palatine unilatérale : tuméfaction qui s'ulcère. Fig. 4. Unilateral palatal NSM: ulcerating mass.

Dans $19 \%$ des cas palatins $[7,8,10,20,27,31,41,48-$ $50,54,57,58,70,89,90,94,95,103]$, la SMN s'est manifestée par une tuméfaction érythémateuse ou recouverte d'une muqueuse saine, évoluant vers la guérison, sans passer par la phase ulcérée. Ces tuméfactions pouvaient être des lésions précoces ou l'expression incomplète de la SMN [61].

La taille des lésions était comprise entre 1,1 et $2,0 \mathrm{~cm}$ dans $32 \%$ des cas palatins $[3-5,8,11,13,17,18,24-26,29,30$, $37,38,40,41,44,56,57,61,63,66,68-70,72,74,75,77$, 78, 81, 84, 86, 88, 90, 92, 93, 95, 102, 105].

La SMN de localisation palatine est devenue destructrice dans $17 \%$ des cas $[3,9,11,12,17,25,29,38,40,41,44$, $50,63-65,69,75,85,104,106]$. En regard de l'ulcération, l'atteinte de l'os sous-jacent pouvait aller d'une lyse osseuse superficielle [64] à une communication bucco-nasale ou buccosinusienne $[25,44,65]$ et l'atteinte des tissus mous à une perforation du voile du palais [106].

\section{SMN des glandes salivaires principales}

On retrouve 22 cas parotidiens $[16,28,42,43,46,111-$ 115], 2 cas submandibulaires $[42,116]$ et 1 cas sublingual [117].

La SMN peut apparaître de novo mais, dans $52 \%$ des cas, elle est survenue dans les jours ou les semaines suivant une intervention chirurgicale locale $[42,43,46] .64 \%$ des cas de SMN étaient associés à une lésion tumorale bénigne ou maligne $[16,28,42,43,46]$; la lésion pouvait également être la conséquence d'une sialadénite lobulaire focale [118].

La SMN se manifestait par une intumescence $[16,42,46$, 111-114, 116, 117], un empâtement localisé de la parotide [115] ou par une sialadénite [42]. Dans un cas, une paralysie faciale périphérique était associée en dehors de tout contexte traumatique ou chirurgical [115]. 


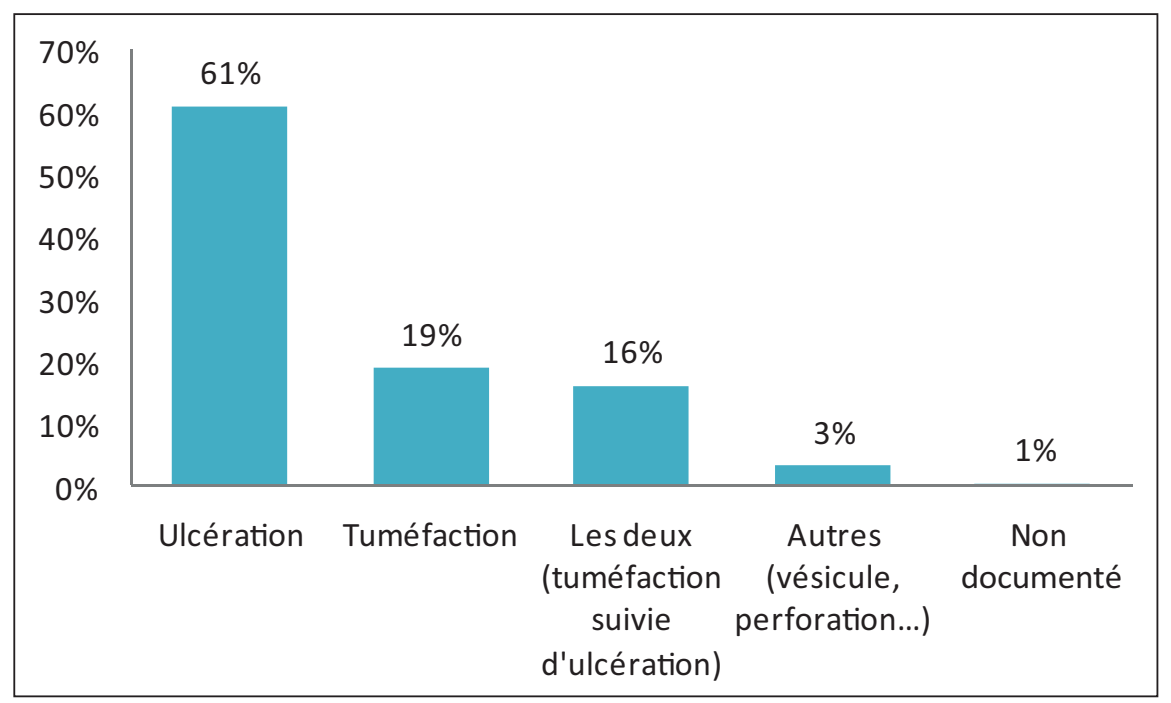

Fig. 5. Répartition des différents aspects cliniques des lésions palatines de SMN. Fig. 5. Distribution of the different palatal lesion clinical aspects of NSM.

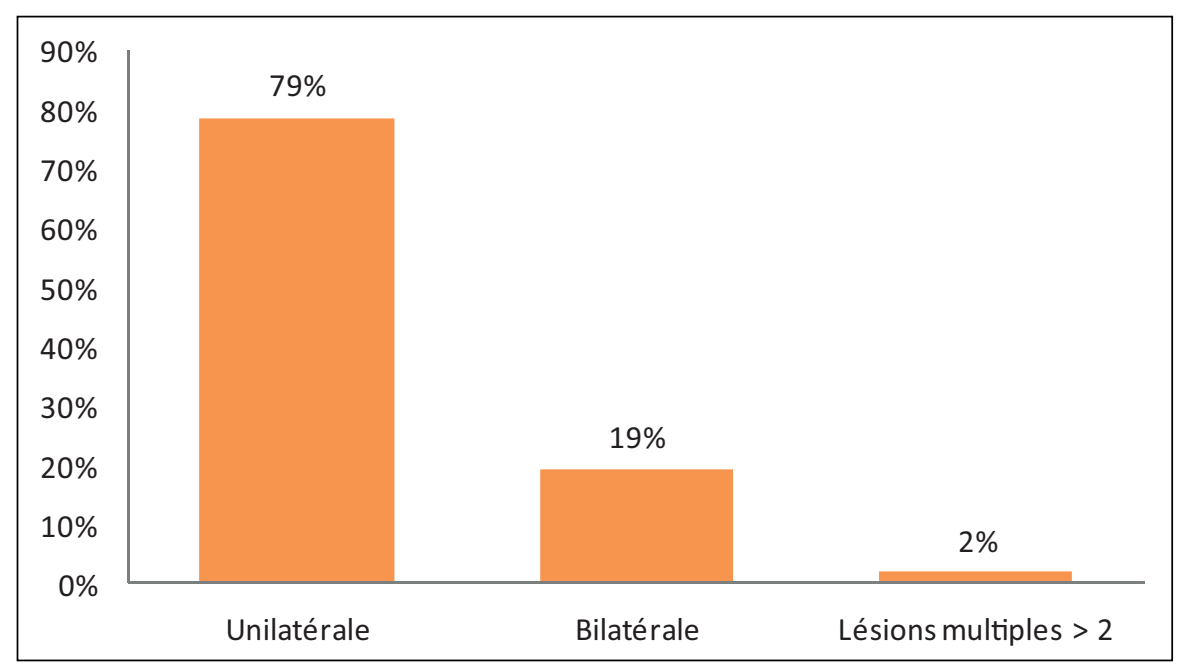

Fig. 6. Répartition du nombre de lésions palatines de SMN.

Fig. 6. Distribution of the palatal lesion number of NSM.

\section{Examen général et signes d'accompagnement de la SMN}

$11 \%$ des cas ont présenté de la fièvre, des migraines ou une adénopathie $[4,5,8,24,29,33,39,58,59,61,62,66$, $68,70,75,80,83,84,89,91,106,115]$. Des signes $0 R L$ ont été retrouvés chez $9 \%$ des cas $[25,29,41,44,57,58,61,65$, 70, 79, 80, 89, 90, 99, 113].

Indolore dans $25 \%$ des cas $[4,8,18,21,22,24,25,27$, $29,31,34,38,40,41,52,56,60,61,63,67,70-72,74,78$, $86,89,93-95,97,100,106-108,112,116,117]$, la SMN était accompagnée de douleurs locales dans $27 \%$ des cas $[5,7,9$, $10,12-15,17,19,24-26,29-33,35-37,39,40,41,48,49$,
$57,61,62,68,70,76,77,81-83,87,88,90,91,93,98,102$, $104,109-111]$, parfois irradiantes vers l'oreille homolatérale ou l'œil ou encore le nasopharynx dans $7 \%$ des cas $[7,8,10$, $41,49,59,64,73,78,85,96,103,105]$. Dans $15 \%$ des cas, elle était associée à des troubles de la sensibilité de la zone concernée ou de la cavité buccale, comme une paresthésie, une hypoesthésie ou une anesthésie locorégionale $[4,7,8,10,12$, $21,24,29,40,52,64,65,70,77,78,82,88-90,92,94,102$, $106,115]$. Il existait parfois des troubles lors de l'alimentation et de la mastication $[8,9,13,17,24,31,36,41,49,57,59$, 68, 69, 84, 89]. 
Tableau II. Diagnostic clinique de la SMN. Table II. NSM's clinical diagnosis.

\begin{tabular}{lcc}
\hline \multicolumn{1}{c}{ DIAGNOSTIC CLINIQUE } & NOMBRE DE CAS $(\boldsymbol{N}=204)$ & POURCENTAGE \\
\hline Diagnostic multiple incluant la SMN & 9 & $5 \%$ \\
Diagnostic multiple n'inculant pas la SMN & 5 & $2 \%$ \\
SMN & 21 & $10 \%$ \\
Adénome pléomorphe & 2 & $1 \%$ \\
Infection (tuberculose, syphilis...) & 4 & $2 \%$ \\
Traumatisme (DDS...) & 5 & $2 \%$ \\
Lésion bénigne & 2 & $1 \%$ \\
Lésion suspecte/maligne & 24 & $12 \%$ \\
Carcinome dont : & 14 & $7 \%$ \\
$\quad$ carcinome épidermoïde & 4 & $1 \%$ \\
$\quad$ carcinome muco-épidermoïde & 4 & $57 \%$ \\
Pathologie granulomateuse & 2 & 116 \\
\hline
\end{tabular}

\section{Diagnostic clinique initial}

La SMN a été diagnostiquée d'emblée dans $10 \%$ des cas $[10,19,25,29,35,40,67,70,77,80,86,101,102]$. Les cliniciens ont évoqué une lésion maligne ou suspecte dans $12 \%$ des cas $[11,16,17,33,37,42,44,46,49,59,60,64,72,73$, $75,104,115]$. Les lésions de SMN ont été diagnostiquées comme carcinomes dans $7 \%$ des cas $[4,7,28,34,36,39,52$, $69,103,108]$. Le diagnostic de SMN pouvait être inclus dans une liste de diagnostics [12, 21, 26, 30, 32, 68, 76, 83, 84] ou en être exclu $[13,28,31,38,94]$. D'autres diagnostics cliniques ont été proposés, à savoir : lésion traumatique $[28,35$, $88,90,110]$, lésion infectieuse $[18,24,37,56]$, adénome pléomorphe $[98,114]$, lésion bénigne $[27,51]$ ou encore pathologie granulomateuse [27, 106]. Dans $57 \%$ des cas, le diagnostic clinique n'était pas précisé $[3-5,7-10,14,15,20-24,29,31$, 40-43, 45, 47, 48, 50, 53-55, 57, 58, 61-63, 65, 66, 70, 71, $74,78,79,81,82,85,87,89-93,95-100,105,107,109,111-$ 113, 116, 117] (Tab. II).

\section{Diagnostic histologique}

Le diagnostic histologique repose sur la présence de 5 critères morphologiques décrits par Abrams et al. [3] :

1) Une nécrose ischémique des lobules des glandes salivaires accessoires.

2) Dans les lobules adjacents, une métaplasie épidermoïde de certains canaux et acini ; cette métaplasie malpighienne peut obstruer totalement la lumière des canaux, les transformant en îlots compacts.
3) Des critères cytonucléaires de bénignité malgré un nombre élevé de mitoses.

4) Dans le tissu conjonctif interstitiel, il existe autour des glandes intéressées une suffusion de mucus et une inflammation réactionnelle. Cet infiltrat subaigu qui siège dans un tissu de granulation, est constitué de lymphocytes, de plasmocytes, d'histiocytes et de polynucléaires neutrophiles.

5) Le respect de l'architecture lobulaire de la glande.

Un an plus tard, Dunlap et al. [4] ont souligné l'existence d'un $6^{\mathrm{e}}$ critère :

6) L'épithélium malpighien de surface, adjacent à l'ulcération, est hyperplasique, papillomateux, sans anomalie cytologique ou architecturale, réalisant une hyperplasie pseudo-épithéliomateuse.

Dans $70 \%$ des cas, les pathologistes ont diagnostiqué une SMN $[3-5,7,8,10,12-16,18,20,21,23,24,27-33,35-43$, 45-60, 62, 64-66, 68, 70, 73, 74, 76-80, 82, 84, 86, 89-91, $96-98,100,102-104,106,110-114,117]$; dans $12 \%$ des cas, un carcinome $[3,4,7,11,20,22,26,27,36,44,57,61,63$, 71, 81, 85, 87, 90, 93, 105, 107, 109].

D'autres diagnostics ont été proposés : hyperplasie pseudoépithéliomateuse [61], hyperplasie papillaire [61], métaplasie épidermoïde [34, 94], fibrose [115], adénome pléomorphe [95], granulomatose de Wegener [101] ou lésion bénigne comme un papillome [17, 29, 50, 93].

Enfin, dans $10 \%$ des cas, aucun diagnostic n'a été proposé. En effet, on comptabilisait $4 \%$ de biopsies non concluantes $[9,29,69,72,75,83,88,92]$ et $6 \%$ de cas où la biopsie n'a 
Tableau III. Diagnostic histologique initial à la biopsie.

Table III. Initial histological diagnosis.

\begin{tabular}{lcc}
\hline \multicolumn{1}{c}{ DIAGNOSTIC HISTOLOGIQUE INITIAL } & NOMBRE DE CAS $(\boldsymbol{N}=\mathbf{2 0 4})$ & POURCENTAGE \\
\hline Hyperplasie pseudo-épithéliomateuse & 1 & $1 \%$ \\
Hyperplasie papillaire & 1 & $1 \%$ \\
Métaplasie épidermoïde & 2 & $1 \%$ \\
Fibrose & 1 & $1 \%$ \\
Lésion bénigne (papillome...) & 4 & $2 \%$ \\
Tumeur mixte (adénome pléomorphe) & 1 & $1 \%$ \\
Carcinome dont : & 25 & $12 \%$ \\
$\quad$ carcinome épidermoïde & 12 & \\
$\quad$ carcinome muco-épidermoïde & 13 & $1 \%$ \\
Granulomatose de Wegener & 1 & $70 \%$ \\
SMN & 145 & $4 \%$ \\
Biopsie non concluante & 10 & $6 \%$ \\
Pas de biopsie & 13 & \\
\hline
\end{tabular}

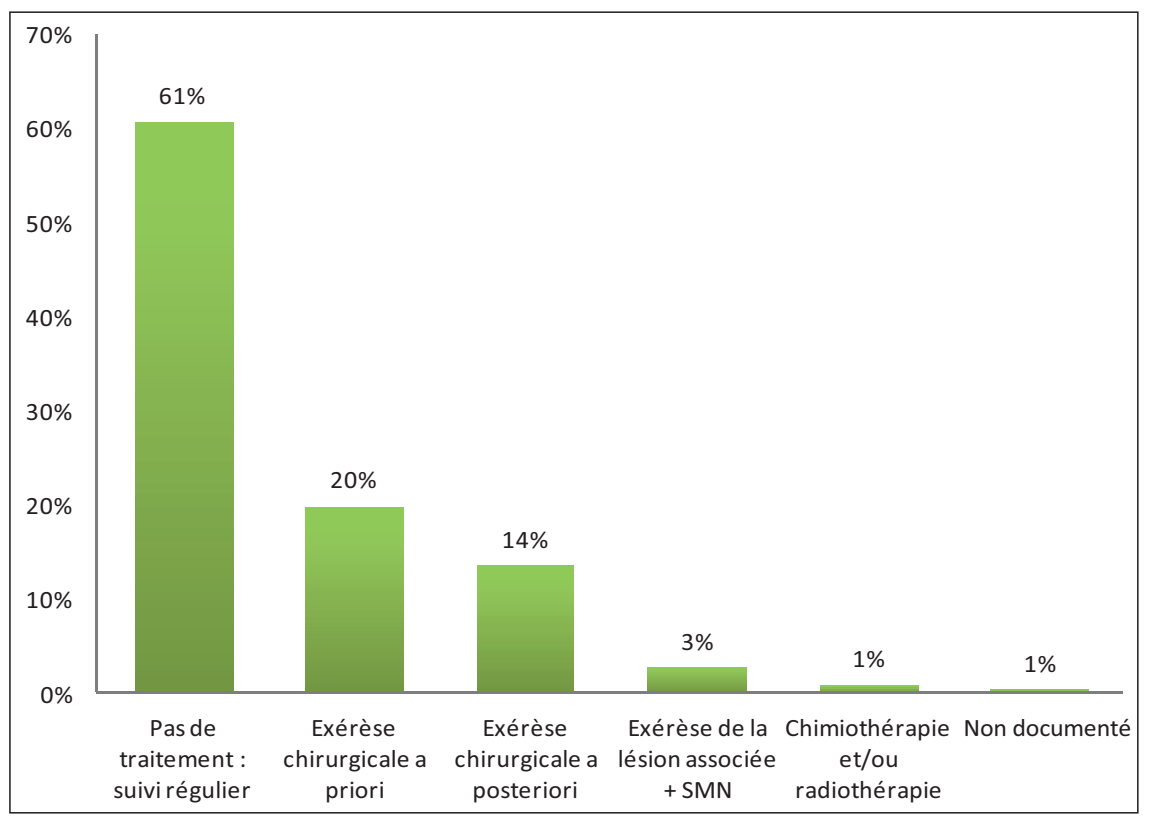

Fig. 7. Répartition des différents traitements de la SMN.

Fig. 7. Distribution of the several SMN's treatment.

pas été réalisée, soit par choix des cliniciens, soit par refus du patient $[19,24,25,67,70,99,108,116]$ (Tab. III).

\section{Traitement}

L'évolution a été spontanément favorable et la durée de la cicatrisation proportionnelle à la taille de la lésion initiale (Fig. 3b). Cette évolution spontanée est un argument diagnostique déterminant en faveur d'une SMN $[3,69,97]$. Un cas de récidive a été rapporté [76].

Le traitement de la SMN consistait en un suivi régulier jusqu'à la guérison dans $61 \%$ des cas $[4,5,7-10,12-15,17$, 19-21, 23, 24, 25, 27-29, 32, 34-41, 44, 49, 50, 52, 56, 57, $61,62,64-66,68-70,73,74,76-80,83,86,87,89-103,106$, 113, 116] (Fig. 7). Un cas d'injection intra-lésionnelle de corticoïdes a été rapporté [89]. 
Les traitements chirurgicaux suivants ont été réalisés:

- Une exérèse chirurgicale a priori, dans $20 \%$ des cas : il s'agit d'exérèses réalisées avant l'établissement d'un diagnostic histologique (biopsies-exérèses, biopsies non concluantes ou ré-excisions de tumeurs) $[10,20,23,30$, $31,33,40-43,47,49-51,53,54,60,75,82,84,108$, $111,112,114,115,117]$.

- Une exérèse chirurgicale a posteriori, dans $14 \%$ des cas : il s'agit d'exérèses réalisées après l'établissement d'un diagnostic histologique $[3,7,11,18,20,22,26,27,57$, $58,63,71,81,85,88,90,104,105,107,109,110]$.

- Une exérèse simultanée de la lésion associée et de la SMN, dans $3 \%$ des cas $[28,45,46,48,50]$.

Un traitement par chimiothérapie et/ou radiothérapie de la lésion associée a été réalisé dans $1 \%$ des cas $[16,55,59]$. Un cas n'est pas bien documenté [67].

Un traitement chirurgical radical a été réalisé dans 15 cas (7\%), allant de la résection complète de l'ulcération à la maxillectomie $[7,11,20,26,27,57,63,71,81,85,90,105$, $107,109]$; le diagnostic de carcinomes épidermoïde ou mucoépidermoïde avait été posé lors de l'examen anatopathologique initial.

\section{Discussion}

Les caractéristiques épidémiologiques, étiologiques, cliniques, histologiques et thérapeutiques de la SMN ont été répertoriées. La revue systématique de la littérature comprenait des rapports de cas et des études rétrospectives de faibles séries. Un nombre important de cas n'étaient pas correctement documentés pour l'ensemble des critères.

La SMN touche en général les glandes salivaires accessoires palatines. Des cas de SMN de localisation atypique ont été rapportés. Une métaplasie malpighienne revêtant les mêmes caractéristiques histologiques que la SMN a été décrite dans les glandes séro-muqueuses des fosses nasales, des sinus [23, 119-123], du larynx [124-126], de la trachée [127-129], des glandes mammaires [130] et des glandes sudorales [131-133]. Bien que les termes d'adénométaplasie et de syringométaplasie soient plus appropriés, car les glandes impliquées ne sont pas des glandes salivaires, le diagnostic de « sialométaplasie » a été retenu dans ces localisations rares. Comme cette terminologie semble abusive, ces cas n'ont pas été répertoriés dans cette revue systématique de la littérature.

Aucune étude n'a rapporté l'incidence et la prévalence exacte de la SMN. En effet, sa fréquence est difficile à évaluer en raison de son évolution torpide et spontanément résolutive, de la fréquente méconnaissance de ce diagnostic et enfin des difficultés d'interprétation anatomopathologique [5, 44]. D'après une étude rétrospective portant sur 10414 biopsies buccales, la SMN représenterait $0,03 \%$ des lésions biopsiées [1].
L'étiopathogénie de la SMN est toujours discutée. La théorie vasculaire est étayée par plusieurs études expérimentales chez l'animal. Les études chez le rat montrent que la ligature des artères et/ou des canaux des glandes salivaires, induit des modifications histologiques similaires à celles de la SMN [134, 135]. Les caractéristiques morphologiques de la SMN ont également été observées au cours d'études expérimentales induisant des tumeurs dans les glandes salivaires du rat après l'injection ou l'implantation de diméthylbenzanthracène [134, $136,137]$. Des auteurs ont observé la présence de métaplasie épidermoïde, accompagnée d'hyperplasie, dans les glandes salivaires muqueuses de la cavité buccale situées dans les champs d'irradiation lors du traitement de carcinomes épidermoïdes de la tête et du cou. Ils ont conclu à un effet précoce de la radiothérapie et non à une inflammation chronique seule [138]. Chez le rat, des injections répétées de solutions d'anesthésique local dans le palais induisent, dans les glandes salivaires, des changements histologiques similaires à ceux observés dans la SMN [139].

L'organisation de la vascularisation des glandes salivaires accessoires du palais renforce la théorie vasculaire. La vascularisation est assurée par l'artère palatine descendante qui émerge à travers le foramen grand palatin et se divise en de nombreuses branches collatérales, dont l'artère grande palatine [42]. Les branches principales de l'artère palatine descendante ont une position latérale et une direction antéropostérieure, et seulement quelques petites branches se dirigent en dedans, vers les zones où la SMN est le plus souvent observée. Il s'agit d'une vascularisation terminale qui est particulièrement vulnérable au niveau du foramen et dans sa région distale. En effet, à ce niveau, tout agent capable de causer une réaction inflammatoire ou une lésion vasculaire crée une hyperpression susceptible d'interrompre la circulation et d'occasionner une ischémie en aval [7, 17, 42, 59]. A côté de la nécrose, une thrombose des artères de moyen calibre a été décrite sur des coupes histologiques $[17,42,53,121]$. Les artères des glandes salivaires principales suivent et accompagnent les subdivisions des canaux excréteurs, de telle façon que chaque lobule salivaire a son propre approvisionnement vasculaire. A l'intérieur des lobules, les territoires vasculaires sont aussi typiquement distincts, chacun ayant une vascularisation terminale. Une perturbation de ce système vasculaire après par exemple une procédure chirurgicale pourrait expliquer la SMN [42].

Les autres étiopathogénies proposées (carence, tabac, allergie, infection) semblent peu probables car rapportées de façon sporadique. L'association à la boulimie serait due à une ischémie secondaire aux efforts effectués pour provoquer le vomissement [24, 78, 81].

Cliniquement, la SMN pouvait être confondue avec différentes lésions. Devant un doute diagnostique quant à la bénignité ou la malignité de la lésion, certains auteurs préconisent une surveillance clinique basée sur des photographies. Les photographies 
devaient être comparées, en recherchant la présence d'un tissu de granulation à la base de l'ulcération, et d'une réépithélialisation des berges. Ces changements, survenant en général 2 semaines après les premières biopsies, étaient, selon les auteurs, une preuve de bénignité. Si aucun signe de guérison n'était observé après 2 semaines de suivi, le diagnostic de SMN semblait alors suspect et il était indispensable de réaliser une nouvelle biopsie des bords de la lésion [69].

La question du diagnostic différentiel clinique se posait en termes variables selon la localisation et les lésions élémentaires. Pour les glandes salivaires accessoires, lorsque la lésion était une ulcération palatine, les diagnostics différentiels principaux étaient une tumeur des glandes salivaires accessoires (carcinomes épidermoïde, muco-épidermoïde, adénoïde kystique...), une lésion traumatique ou encore une gomme syphilitique ou tuberculeuse ulcérée. Lorsque la SMN se présentait sous la forme d'une tuméfaction palatine, on évoquait entre autres un torus palatin ou une exostose, une tumeur des glandes salivaires accessoires (carcinomes muco-épidermoïde ou adénoïde kystique, adénocarcinome, adénome pléomorphe...), un sarcome, un lymphome non hodgkinien, un abcès sous-périosté d'origine dentaire ou encore une gomme syphilitique ou tuberculeuse $[32,37,140]$. Pour les glandes salivaires principales, la SMN se présentait comme une tuméfaction nodulaire donnant à la palpation une sensation d'empâtement localisé faisant évoquer différents diagnostics comme les tumeurs parotidiennes (adénome pléomorphe, tumeur de Whartin, carcinome muco-épidermoïde, carcinome adénoïde kystique...), les kystes salivaires, la parotidite tuberculeuse nodulaire $[42,112,113$, 115-117]. L'association d'une paralysie faciale a une SMN parotidienne a fait suspecter une lésion maligne [115].

Histologiquement, la SMN peut faire évoquer différentes lésions bénignes ou malignes. Trois critères permettant de distinguer la SMN des processus malins ont été défini : conservation de l'architecture lobulaire, absence d'atypie cytonucléaire dans les îlots cellulaires et présence d'une lumière canalaire résiduelle dans un ou plusieurs de ces îlots $[3,46]$. Les diagnostics différentiels principaux étaient les carcinomes épidermoïde et muco-épidermoïde. L'existence d'une hyperplasie pseudo-épithéliomateuse de l'épithélium de surface et d'une métaplasie malpighienne extensive peut conduire au diagnostic erroné d'un carcinome épidermoïde originaire de l'épithélium de surface infiltrant le tissu glandulaire, ou d'un carcinome muco-épidermoïde venant au contact de l'épithélium de surface, lorsqu'il persiste quelques îlots de cellules mucosécrétantes [32, 37, 44]. Il est donc indispensable de :

- faire des biopsies assez larges et profondes, à cheval sur l'ulcération et une de ses berges, afin de confirmer la persistance de l'architecture glandulaire qui n'était souvent visible qu'à un faible grossissement [3],

- pratiquer de nombreux plans de coupe pour l'observation histologique,
- réaliser une confrontation anatomoclinique de qualité, précisant la durée et le mode d'évolution de cette lésion [44].

La difficulté du diagnostic histologique de la SMN s'exprimait par un pourcentage important $(12 \%)$ de diagnostics erronés de carcinomes. Ceci pouvait aboutir à une thérapeutique radicale délabrante $(7 \%)$, comme dans un cas de SMN de la cavité nasale [122]. Une maxillectomie a été effectuée après que le diagnostic erroné de carcinome épidermoïde ait été posé. L'examen histologique de la pièce d'exérèse chirurgicale $n^{\prime}$ avait retrouvé aucun signe de malignité. Le diagnostic de SMN avait été retenu après réexamen anatomopathologique du prélèvement initial.

L'intérêt de la corticothérapie dans le traitement de la SMN a été discuté. Un cas d'injection intra-lésionnelle de corticoïdes a été rapporté [89]. Il s'agissait de tuméfactions bilatérales palatines, les corticoïdes avaient été injectés localement dans l'une des tuméfactions, la seconde tuméfaction étant le témoin. Les auteurs ont constaté une absence de réponse, qui reflétait probablement, selon eux, le fait que le processus nécrotique était complet avant le début du traitement. Instaurer un traitement plus précocement, était difficile cliniquement en raison du délai plus ou moins important entre la découverte et le diagnostic de la lésion.

\section{Conclusion}

La SMN est une affection bénigne rare des glandes salivaires accessoires de diagnostic parfois difficile. Elle survient essentiellement chez l'adulte et se manifeste typiquement par une tuméfaction ou une ulcération palatine d'apparition brutale.

L'intérêt de sa connaissance réside dans le risque de confusion anatomoclinique avec certaines lésions néoplasiques pouvant conduire à des traitements délabrants. Pour illustrer son apparence trompeuse, des auteurs la décrivent même comme « a sheep in wolf's clothing » $[69,141]$.

En raison des difficultés d'interprétation anatomopathologiques, il semble indispensable de faire des biopsies de grande taille, à cheval sur l'ulcération et une de ses berges, de pratiquer de nombreux plans de coupe et de réaliser une confrontation anatomoclinique de qualité.

Enfin, la certitude du diagnostic n'est apportée que par l'évolution spontanément résolutive de la lésion, la cicatrisation survenant dans les 3 à 10 semaines suivant l'apparition de l'ulcération [142, 143].

\section{Conflits d'intérêt : aucun}

\section{Références}

1. Mesa ML, Gertler RS, Schneider LC. Necrotizing sialometaplasia: frequency of histologic misdiagnosis. Oral Surg Oral Med Oral Pathol 1984;57:71-3. 
2. Saunders WH. Nicotine stomatitis of the palate. Ann Otol Rhinol Laryngol 1958;67:618-27.

3. Abrams AM, Melrose RJ, Howell FV. Necrotizing sialometaplasia. A disease simulating malignancy. Cancer 1973;32:130-5.

4. Dunlap CL, Barker BF. Necrotizing sialometaplasia. Report of five additional cases. Oral Surg Oral Med Oral Pathol 1974;37:722-7.

5. Brocheriou C, Bertrand JC. Sialométaplasie nécrosante. Une observation. Rev Stomatol Chir Maxillofac 1979;80:359-62.

6. Brannon RB, Fowler CB, Hartman KS. Necrotizing sialometaplasia. A clinicopathologic study of sixty-nine cases and review of the literature. Oral Surg Oral Med Oral Pathol 1991;72:317-25.

7. Arguelles MT, Viloria JB Jr, Talens MC, McCory TP. Necrotizing sialometaplasia. Oral Surg Oral Med Oral Pathol 1976;42:86-90.

8. Gahhos F, Enriquez RE, Bahn SL, Ariyan S. Necrotizing sialometaplasia: report of five cases. Plast Reconstr Surg 1983;71:650-7.

9. Dunley RE, Jacoway JR. Necrotizing sialometaplasia. Oral Surg Oral Med Oral Pathol 1979;47:169-72.

10. Femopase FL, Hernandez SL, Gendelman H, Criscuolo MI, Lopez De Blanc SA. Sialometaplasia necrotizante: presentación de cinco casos clinicos. Med Oral 2004;9:304-8.

11. Mitchell RD. Necrotizing sialometaplasia. Case report. Aust Dent J 1985;30:181-4.

12. Newland J. Bilateral presentation of necrotizing sialometaplasia - a case report. Dent Update 2007;34:586-8.

13. Raugi GJ, Kessler S. Necrotizing sialometaplasia. A condition simulating malignancy. Arch Dermatol 1979;115:329-31.

14. Spark RP, Duncan DG. Necrotizing sialometaplasia. A self-limited pseudotumoral palatal ulcer. Ann Otol Rhinol Laryngol 1978;87:409-11.

15. Stühmer C, Essig H, Feist H, Bormann KH, Gellrich NC, Rücker M. Die nekrotisierende sialometaplasie: diagnostik einer seltenen entität. Schweiz Monatsschr Zahnmed 2008;118:635-40.

16. Yoshioka T, Harada M, Umekita Y, Taguchi S, Higashi M, Nakamura D, Suzuki S, Tanimoto A. Necrotizing sialometaplasia of the parotid gland associated with angiocentric T-cell lymphoma: a case report and review of the literature. Pathol Int 2010;60:326-9.

17. Rye LA, Calhoun NR, Redman RS. Necrotizing sialometaplasia in a patient with Buerger's disease and Raynaud's phenomenon. Oral Surg Oral Med Oral Pathol 1980;49:233-6.

18. Bannayan G, Fox G, Tilson HB. Necrotizing sialometaplasia of the palate. J Oral Surg 1976;34:727-30.

19. García CM, Gil FM, Gil HS, Maestre 0, Alcohol LV, Laza LR, Sánchez AJM, Zaldivar DMSD. Sialometaplasia necrotizante. Rev Esp Cir Oral Maxilofac 2008;30:286-90.

20. Grillon GL, Lally ET. Necrotizing sialometaplasia: literature review and presentation of five cases. J Oral Surg 1981;39:747-53.

21. Imbery $T A$, Edwards PA. Necrotizing sialometaplasia: literature review and case reports. J Am Dent Assoc 1996;127:1087-92.

22. Sandmeier D, Bouzourene H. Necrotizing sialometaplasia: a potential diagnostic pitfall. Histopathology 2002;40:200-1.

23. Van der Wal JE, Van der Waal I. Necrotizing sialometaplasia: report of 12 new cases. Br J Oral Maxillofac Surg 1990;28:326-8.
24. Boisnic S, Agbo S, Biaggi A, Pascal F, Szpirglas H, Frances C. La sialométaplasie nécrosante du palais: 3 observations. Ann Dermatol Venereol 1992;119:922-4.

25. Bertoin P, Baudet-Pommel M, Devoize L. Sialométaplasie nécrosante. A propos de 6 cas et revue de littérature. Clinic 2002;23:607-12.

26. Birkholz H, Brownd CL. Necrotizing sialometaplasia: report of an ulcerative case. J Am Dent Assoc 1981;103:48-50.

27. Dominguez FV, Espinal EG. Sialométaplasie nécrosante. Présentation de deux cas et revue de la littérature. Rev Odontostomatol 1982;11:375-8.

28. Favia G, Pratelli D, Maiorano E. La sialometaplasia necrotizzante. Analisi clinico-patologica di 6 casi. Minerva Stomatol 1996;45:121-7.

29. Gavron JP, Ardito JA, Curtis AW. Necrotizing sialometaplasia. Laryngoscope 1981;91:1176-80.

30. Gavron JP, Shugar MA, Rice DA. Necrotizing sialometaplasia. Am Fam Physician 1983;27:155-7.

31. Giles AD. Necrotizing sialometaplasia. Br J Oral Surg 1980;18:45-50.

32. Houston G. Oral pathology. Fall 1998 case of the month. Necrotizing sialometaplasia. J Okla Dent Assoc 1998;89:15-9.

33. Hovinga J, De Jager $H$. A patient with necrotizing sialometaplasia. Int J Oral Surg 1977;6:280-2.

34. Kominek P, Blasch P. Necrotizing sialometaplasia: a potential diagnostic pitfall. Ear Nose Throat J 2006;85:604-5.

35. Lambert PM. Necrotizing sialometaplasia: report of two cases. Spec Care Dentist 1987;7:78-80.

36. Lynch DP, Crago CA, Martinez MG Jr. Necrotizing sialometaplasia. A review of the literature and report of two additional cases. Oral Surg Oral Med Oral Pathol 1979;47:63-9.

37. Murphy J, Giunta J, Meyer I, Robinson K. Necrotizing sialometaplasia. Oral Surg Oral Med Oral Pathol 1977;44:419-24.

38. Piette F, Sauque E, Pellerin P, Bergoend H. Sialométaplasie nécrosante. Ann Dermatol Venereol 1980;107:821-4.

39. Silva AD, Silva CA, Furuse C, Nunes e Souza RC, da Costa MH, de Araújo VC. Necrotizing sialometaplasia in a patient who is HIV positive: a case report. Spec Care Dentist 2010;30:160-2.

40. Abaza NA, Abaza MM, Sedhom AW. Necrotizing sialometaplasia: a review and two case reports. Compendium 1992;13:12,14,168 passim.

41. Anneroth G, Hansen LS. Necrotizing sialometaplasia. The relationship of its pathogenesis to its clinical characteristics. Int J Oral Surg 1982;11:283-91.

42. Batsakis JG, Manning JT. Necrotizing sialometaplasia of major salivary glands. J Laryngol Otol 1987;101:962-6.

43. Donath K. Pathohistologie des parotisinfarktes (necrotizing sialometaplasia). Laryngol Rhinol Otol 1979;58:70-6.

44. Dubus J, Andrieu-Guitrancourt J, Beuvon F, Dehesdin D, Thomine E. La sialométaplasie nécrosante: trop méconnue. Acta Otorhinolaryngol Belg 1993;47:355-58.

45. Franchi A, Gallo 0, Santucci M. Pathologic quiz case 1. Necrotizing sialometaplasia obscuring recurrent well-differentiated squamous cell carcinoma of the maxillary sinus. Arch Otolaryngol Head Neck Surg 1995;121:584-6. 
46. Gnepp DR. Warthin tumor exhibiting sebaceous differentiation and necrotizing sialometaplasia. Virchows Arch A Pathol Anat Histol 1981;391:267-73.

47. Granick MS, Solomon MP, Benedetto AV, Hannegan MW, Sohn M. Necrotizing sialometaplasia masquerading as residual cancer of the lip. Ann Plast Surg 1988;21:152-4.

48. Lee DJ, Ahn HK, Koh ES, Rho YS, Chu HR. Necrotizing sialometaplasia accompanied by adenoid cystic carcinoma on the soft palate. Clin Exp Otorhinolaryngol 2009;2:48-51.

49. Nilsen $\mathrm{R}$, Bernhoft $\mathrm{CH}$, Gilhuus-Moe 0. Necrotizing sialometaplasia. Int J Oral Surg 1978;7:580-4.

50. Pabuççuoglu U, Özkara E, Sarioglu S. Necrotizing sialometaplasia. Report of five cases including uncommon localizations. Turk J Med Sci 2002;32:355-9.

51. Pulse CL, Lebovics RS, Zegarelli DJ. Necrotizing sialometaplasia: report of a case after lower lip mucocele excision. J Oral Maxillofac Surg 2000;58:1419-21.

52. Randhawa T, Varghese I, Shameena PM, Sudha S, Nair RG. Necrotizing sialometaplasia of tongue. J Oral Maxillofac Pathol 2009;13:35-7.

53. Willen $H$, Willen R, Ekman L. Necrotizing sialometaplasia of the bucca. Acta Pathol Microbiol Scand A 1981;89:199-201.

54. Ylikontiola L, Siponen M, Salo T, Sandor GK. Sialometaplasia of the soft palate in a 2-year-old girl. J Can Dent Assoc 2007;73:333-6.

55. Dominguez-Malagon H, Mosqueda-Taylor A, Cano-Valdez AM. Necrotizing sialometaplasia of the palate associated with angiocentric T-cell lymphoma. Ann Diagn Pathol 2009;13:60-4.

56. Krishna S, Bk R. Necrotizing sialometaplasia of palate: a case report. Imaging Sci Dent 2011;41:35-8.

57. Marciani RD, Sabes WR. Necrotizing sialometaplasia: report of three cases. J Oral Surg 1976;34:722-6.

58. Niedzielska I, Janic T, Markowski J. Bilateral localization of necrotizing sialometaplasia: a case report. Cases J 2009;2:9068.

59. Poulson TC, Greer RO Jr, Ryser RW. Necrotizing sialometaplasia obscuring an underlying malignancy: report of a case. J Oral Maxillofac Surg 1986;44:570-4.

60. Reychler H, Berger PE, Dourov N. Tumeur d'Abrikossof de la langue associée à une lésion de sialométaplasie. Rev Stomatol Chir Maxillofac 1983;84:210-7.

61. Samit AM, Mashberg A, Greene GW Jr. Necrotizing sialometaplasia. J Oral Surg 1979;37:353-6.

62. Bascones-Martínez A, Muñoz-Corcuera M, Cerero-Lapiedra R, Bascones-Ilundain J, Esparza-Gomez G. Case report of necrotizing sialometaplasia. Med Oral Patol Oral Cir Buccal 2011;16:e700-3.

63. Chakravorty RC, Yoneyama T, Makooi C. Necrotizing sialometaplasia of palate. Br J Surg 1979;66:283-4.

64. Constantini L, Balaton A, Princ G. Sialométaplasie nécrosante. A propos d'un cas. Problèmes diagnostique et thérapeutique. Rev Stomatol Chir Maxillofac 1988;89:234-6.

65. Daudia A, Murty GE. First case of full-thickness palatal necrotizing sialometaplasia. J Laryngol Otol 2002;116:219-20.

66. Fava M, Cherubini K, Yurgel L, Salum F, Figueiredo MA. Necrotizing sialometaplasia of the palate in a cocaine-using patient. Minerva Stomatol 2008;57:199-202.
67. Gordon S, Geist RY, Hirschman B, Geist J. Oral pathology quiz. Case 1. Necrotizing sialometaplasia. J Mich Dent Assoc 2001;83:36-8.

68. Johann AC, Aguiar MC, Mesquita RA, Vieira do Carmo MA. Necrotizing sialometaplasia of the palate. Oral Oncol Extra 2006;42:147-9.

69. Kinney RB, Burton CS, Vollmer RT. Necrotizing sialometaplasia: a sheep in wolf's clothing. Arch Dermatol 1986; 22:208-210.

70. Lamey PJ, Lewis MA, Crawford DJ, MacDonald DG. Necrotising sialometaplasia presenting as greater palatine nerve anaesthesia. Int J Oral Maxillofac Surg 1989;18:70-2.

71. Matilla A, Flores T, Nogales FF Jr, Galera H. Necrotizing sialometaplasia affecting the minor labial glands. Oral Surg Oral Med Oral Pathol 1979;47:161-3.

72. Merwin GE, Duckert LG, Pollak K. Necrotizing sialometaplasia of the nasopharynx. Ann Otol Rhinol Laryngol 1979;88:348-51.

73. Philipsen HP, Petersen JK, Simonsen BH. Necrotizing sialometaplasia of the palate. Ulcerative or necrotizing stage of leukokeratosis nicotina palati? Int J Oral Surg 1976;5:292-9.

74. Rapisarda F, Ficarra G. Sialometaplasia necrotizzante. Presentazione di un caso. Minerva Stomatol 1999;48:273-5.

75. Rolland A, Martin H, Lebeau J, Antoine P, Raphael B. La sialométaplasie nécrosante. Un piège diagnostique rare. Rev Stomatol Chir Maxillofac 1983;84:359-62.

76. Rossie KM, Allen CM, Burns RA. Necrotizing sialometaplasia: a case with metachronous lesions. J Oral Maxillofac Surg 1986;44:1006-8.

77. Santis HR, Kabani SP, Roderiques A, Driscoll JM. Necrotizing sialometaplasia: an early, nonulcerative presentation. Oral Surg Oral Med Oral Pathol 1982;53:387-90.

78. Schöning H, Emshoff R, Kreczy A. Necrotizing sialometaplasia in two patients with bulimia and chronic vomiting. Int $\mathrm{J}$ Oral Maxillofac Surg 1998;27:463-5.

79. Schroeder WA Jr. Necrotizing sialometaplasia. Otolaryngol Head Neck Surg 1994;111:328-9.

80. Scully C, Eveson J. Sialosis and necrotizing sialometaplasia in bulimia; a case report. Int J Oral Maxillofac Surg 2004;33:808-10.

81. Solomon LW, Merzianu M, Sullivan M, Rigual NR. Necrotizing sialometaplasia associated with bulimia: case report and literature review. Oral Surg Oral Med Oral Pathol Oral Radiol Endod 2007;103:e39-42.

82. Sortino F. La scialometaplasia necrotizzante. Caso clinico. Minerva Stomatol 2002;51:213-7.

83. Stafford RF, Sonis ST, Shklar G. Bilateral necrotizing sialometaplasia: a case report. J Oral Med 1981;36:28-30.

84. Tinoco PJ, Salazar N. Sialometaplasia necrotizante pseudoepiteliomatosa. Recuento de la literature, presentación de un caso y modificación a su nomenclatura. Acta Odontol Venez 1984;22:9-27.

85. Viala I, Stierle JL, Collange S, Hemar P, Conraux C, Bellocq JP, Chenard MP. Sialométaplasie nécrosante: un piège diagnostique. J Fr Otorhinolaryngol 1997;46:321-3.

86. Vittori F, Caudmont M, Pham B. Sialométaplasie nécrosante au cours d'un SIDA. Sem Hop Paris 1994;70:98-9.

87. Wills PI, Fechner RE. Necrotizing sialometaplasia. Pathologic quiz case 1. Arch Otolaryngol 1975;101:76-8. 
88. Williams RF. Necrotizing sialometaplasia after bronchoscopy. J Oral Surg 1979;37:816-8.

89. Keogh PV, O'Regan E, Toner M. Flint S. Necrotizing sialometaplasia: an unusual bilateral presentation associated with antecedent anaesthesia and lack of response to intralesional steroids. Case report and review of the literature. $\mathrm{Br}$ Dent $\mathrm{J}$ 2004;196:79-81.

90. Suckiel JM, Davis WH, Patakas BM, Kaminishi RM. Early and late manifestations of necrotizing sialometaplasia. J Oral Surg 1978;36:902-5.

91. Toftegaard Funding A, Steinicke T, Iversen L. Necrotizing sialometaplasia: a case report. Acta Derm Venereol 2004;84:403-4.

92. Piquet JJ, Darras JA, Burny A. Sialométaplasie nécrosante. J Fr Otorhinolaryngol 1985;34:361-3.

93. Aknin J, Dubreuil C, Seguin P. La sialométaplasie nécrosante. A propos de deux observations. J Fr Otorhinolaryngol 1989;38:188-92.

94. Birkholz H, Minton GA, Yuen YL. Necrotizing sialometaplasia: review of the literature and report of nonulcerative case. $J$ Oral Surg 1979;37:588-92.

95. Buller DL. Nodular and ulcerated lesions of hard palate. J Am Dent Assoc 1980;101:823-4.

96. Capelli M, Caputo V, Saginario V, Susicky A, Castellani C, Jankovic C, Berardi A, La Guardia M, Berardi C. A clinical case of necrotizing sialometaplasia and conjunctival hemorrhage. Minerva Stomatol 2010;59:437-43.

97. Cavelier-Balloy B, Chemaly P, Laufer J, Brocheriou C, Civatte J. Sialométaplasie nécrosante. Ann Dermatol Venereol 1984;111:679-80.

98. Chaudhry AP, Yamane GM, Salman L. Salman S, Saxon M, Pierri LK. Necrotizing sialometaplasia of palatal minor salivary glands: a report on 2 cases. J Oral Med 1985;40:2-6.

99. Crumpton B, McGinnis JP, Krolls S0. Determining the nature of oral ulcerations. Dentistry 1998;18:24-5.

100. De Saint Aubain Somerhausen N, Larsimont D, Tant L. Verhest A. La sialométaplasie nécrosante : à propos d'une observation et revue de la littérature. Acta Stomatol Belg 1996;93:61-3.

101. Farina D, Gavazzi E, Avigo C, Borghesi A, Maroldi R. MRI findings of necrotizing sialometaplasia. Br J Radiol 2008;81:e173-5.

102. Mandel L, Kaynar A, DeChiara S. Necrotizing sialometaplasia in a patient with sickle-cell anemia. J Oral Maxillofac Surg 1991;49:757-9.

103. Oliveira Alves MG, Kitakawa D, Carvalho YR, Guimaraes Cabral LA, Almeida JD. Necrotizing sialometaplasia as a cause of a nonulcerated nodule in the hard palate: a case report. J Med Case Reports 2011;5:406.

104. Bell GW, Loukota RA. Necrotizing sialometaplasia coincident with ipsilateral infarcted antral polyps. Br J Oral Maxillofac Surg 1996;34:129-31.

105. Myers EN, Bankaci M, Barnes EL Jr. Necrotizing sialometaplasia. Report of a case. Arch Otolaryngol 1975;101:628-9.

106. Speechley JA, Field EA, Scott J. Necrotizing sialometaplasia occurring during pregnancy: report of a case. J Oral Maxillofac Surg 1988;46:696-9.

107. Fechner RE. Necrotizing sialometaplasia. A source of confusion with carcinoma of the palate. Am J Clin Pathol 1977;67:315-7.
108. Papanayotou PH, Kayavis JG, Epivatianos AA, Trigonidis G. Necrotizing sialometaplasia of the cheek: report of case and review of literature. J Oral Surg 1980;38:538-40.

109. Anneroth G, Bystedt H, Hammarström L. Necrotizing sialometaplasia-a malignancy simulating oral lesion. Swed Dent J 1986;10:53-8.

110. Forney SK, Foley JM, Sugg WE Jr, Oatis GW Jr. Necrotizing sialometaplasia of the mandibule. Oral Surg Oral Med Oral Pathol 1977;43:720-6.

111. Aydin 0, Yilmaz T, Ozer F, Saraç S, Sökmensüer C. Necrotizing sialometaplasia of parotid gland: a possible vasculitic cause. Int J Pediatr Otorhinolaryngol 2002;64:171-4.

112. Nemecek JR, Marzek PA, Young VL. Diagnosis and treatment of accessory parotid gland masses. Ann Plast Surg 1994;33:75-9.

113. Prabhakaran VC, Flora RS, Kendall C. Pressure-induced necrotizing sialometaplasia of the parotid gland. Histopathology 2006;48:464-5.

114. Sarioglu S, Pabuççuoglu U, Ecevit C, Ceryan K, Paksoy S, Ada E. Sialometaplasia arising in the ectopic salivary gland ductal inclusions of multiple intraparotid lymph nodes. J Clin Pathol 2004;57:1335-7.

115. Stoll D, Chambrin H, Auria JP, Deminière C. La sialométaplasie nécrosante aiguë de la parotide. Rev Laryngol Otol Rhinol 1991;112:171-2.

116. Russo A, Dell'Aquila A, Prota V, Sica GS. Scialometaplasia necrotizzante della ghiandola sottomandibolare. Descrizione di un caso. Minerva Stomatol 1998;47:273-7.

117. Lima MA, Rocha LC, Siqueira LM, Carmo LC, Filho JH. Forma cística da sialomataplasia necrosante em glândula salivar sublingual. Rev Bras Otorrinolaringol 2002;68:276-9.

118. Batsakis JG, Regezi JA. Selected controversial lesions of salivary tissues. Otolaryngol Clinics North Am 1977;10:309-27.

119. Chen KT. Necrotizing sialometaplasia of the nasal cavity. Am J Otolaryngol 1982;3:444-6.

120. Granich MS, Pilch BZ. Necrotizing sialometaplasia in the setting of acute and chronic sinusitis. Laryngoscope 1981;91:1532-5.

121. Johnston WH. Necrotizing sialometaplasia involving the mucous glands of the nasal cavity. Hum Pathol 1977;8:589-92.

122. Maisel RH, Johnston WH, Anderson HA, Cantrell RW. Necrotizing sialometaplasia involving the nasal cavity. Laryngoscope 1977;87:429-34.

123. Russel JD, Glover GW, Friedmann I. Necrotizing sialometaplasia. J Laryngol Otol 1992;106:569-71.

124. Ravn T, Trolle W, Kiss K, Balle VH. Adenosquamous carcinoma of the larynx associated with necrotizing sialometaplasia-a diagnostic challenge. Auris Nasus Larynx 2009;36:721-4.

125. Walker GK, Fechner RE, Johns ME, Teja K. Necrotizing sialometaplasia of the larynx secondary to atheromatous embolization. Am J Clin Pathol 1982;77:221-3.

126. Wenig BM. Necrotizing sialometaplasia of the larynx. A report of two cases and a review of the literature. Am J Clin Pathol 1995;103:609-13.

127. Ben-Izhak 0, Ben-Arieh Y. Necrotizing squamous metaplasia in herpetic tracheitis following prolonged intubation: a lesion similar to necrotizing sialometaplasia. Histopathology 1993;22:265-9. 
128. Littman CD. Necrotizing sialometaplasia (adenometaplasia) of the trachea. Histopathology 1993;22:298-9.

129. Romagosa V, Bella MR, Truchero C, Moya J. Necrotizing sialometaplasia (adenometaplasia) of the trachea. Histopathology 1992;21:280-2.

130. Hurt MA, Diaz-Arias AA, Rosenholtz MJ, Havey AD, Stephenson HE Jr. Posttraumatic lobular squamous metaplasia of breast. An unusual pseudocarcinomatous metaplasia resembling squamous (necrotizing) sialometaplasia of the salivary gland. Mod Pathol 1988;1:385-90.

131. Hurt MA, Halvorson RD, Petr FC, Cooper JT Jr, Friedman DJ. Eccrine squamous syringometaplasia. A cutaneous sweat gland reaction in the histologic spectrum of 'chemotherapy-associated eccrine hidradenitis' and 'neutrophilic eccrine hidradenitis'. Arch Dermatol 1990;126:73-7.

132. King DT, Barr RJ. Syringometaplasia: mucinous and squamous variants. J Cutan Pathol 1979;6:284-91.

133. Metcalf JS, Maize JC. Squamous syringometaplasia in lobular panniculitis and pyoderma gangrenosum. Am J Dermatopathol 1990;12:141-9.

134. Englander A, Cataldo E. Experimental carcinogenesis in duct-artery ligated rat submandibular gland. J Dent Res 1976;55:229-34.

135. Standish SM, Shafer WG. Serial histologic effects of rat submaxillary and sublingual salivary gland duct and blood vessel ligation. J Dent Res 1957;36:866-79.
136. Cataldo E, Shklar G. Experimental salivary gland tumors in rats. Arch Pathol 1969;77:305-16.

137. Glucksman A, Cherry CP. The effect of sex and of sex and thyroid hormones on the induction of cancers in the salivary glands of rats. Br J Cancer 1966;20:760-77.

138. Friedman M, Hall JW. Radiation induced squamous cell metaplasia and hypertrophy of the normal mucous glands of the oral cavity. Radiology 1950;55:848-51.

139. Shigematsu H, Shigematsu Y, Noguchi Y, Fujita K. Experimental study on necrotizing sialometaplasia of the palate in rats. Role of local anesthetic injections. Int J Oral Maxillofac Surg 1996;25:239-41.

140. Boutremans E, Shahla M, Tant L, Javadian R, De Saint Aubain N, Loeb I. A propos d'une ulcération palatine. Rev Stomatol Chir Maxillofac 2006;107:179-80.

141. Samson J, El Hage M, Carrel JP. A sheep in wolf's clothing. Med Buccale Chir Buccale 2010;16:57.

142. Damardji R. Sialométaplasie nécrosante : présentation de 9 cas. Thèse de doctorat en médecine dentaire. Université de Genève, 2011, $n^{\circ} 692$.

143. Samson J, Lysitsa S, Abi Najm S, Carrel JP. La sialométaplasie nécrosante. Alpha Omega News 2003;104:6-9. 